Tropical Journal of Pharmaceutical Research April 2021; 20 (4): 789-796

ISSN: $1596-5996$ (print); 1596-9827 (electronic)

(C) Pharmacotherapy Group, Faculty of Pharmacy, University of Benin, Benin City, 300001 Nigeria.

\title{
Mechanism of pharmacological effect of Angelica essential oil on anxiety based on network pharmacology and molecular docking
}

\author{
Xiaoli Liu1, Xiaofei Zhang ${ }^{2,3}$, Fang Wang ${ }^{3}$, Jie $\mathrm{Xu}^{3}$, Ming Yang ${ }^{1,3 *}$ \\ ${ }^{1}$ School of Pharmacy, State Key Laboratory of Southwestern Chinese Medicine Resources, Chengdu University of Traditional \\ Chinese Medicine, Chengdu City, Sichuan Province 611137, ²Pharmacy College, Shaanxi University of Chinese Medicine, \\ Xianyang City, Shaanxi Province $712046,{ }^{3}$ Key Laboratory of Modern preparation of Traditional Chinese Medicine, Ministry of \\ Education, Jiangxi University of Traditional Chinese Medicine, Nanchang City, Jiangxi 330000, China
}

*For correspondence: Email: Mingyang26@126.com; Tel: +86-0791-87118658

Sent for review: 12 November 2020

Revised accepted: 20 March 2021

\begin{abstract}
Purpose: To investigate the pharmacological effect of Angelica essential oil (AEO) on anxiety disorder and the mechanism of action involved, using network pharmacology and molecular docking.

Method: Chemical compositions of AEO were determined using gas chromatography-mass spectrometry (GC-MS). The potential targets involved in the effect of AEO on anxiety were predicted by searching Bioinformatics databases. Cytoscape software was applied for networks construction. Analysis of Gene Ontology (GO) and pathway was done via R Programming Language, while molecular docking analysis was conducted by Autodock vina software.

Results: Network analysis showed that there were 10 bioactive components and 34 potential targets. The potential targets were distributed in cerebellar cortex, basic ganglia, hippocampal formation, amygdala, pons and medulla and liver. AEO was involved in multiple biological processes (BP), cell components (CC) and molecular functions (MF) associated with anxiety disorder. Molecular docking revealed good binding affinity between the major bioactive components and targets.

Conclusion: This study has revealed the pharmacological mechanism involved in the effect of AEO on anxiety, through network pharmacology and molecular docking. The pharmacological mechanism provides a firm theoretical basis for further studies in this area of research.
\end{abstract}

Keywords: Angelica Essential oil, Anxiety, Network pharmacology, Molecular Docking, Pharmacological

\begin{abstract}
This is an Open Access article that uses a fund-ing model which does not charge readers or their institutions for access and distributed under the terms of the Creative Commons Attribution License (http://creativecommons.org/licenses/by/4.0) and the Budapest Open Access Initiative (http://www.budapestopenaccessinitiative.org/read), which permit unrestricted use, distribution, and reproduction in any medium, provided the original work is properly credited.
\end{abstract}

Tropical Journal of Pharmaceutical Research is indexed by Science Citation Index (SciSearch), Scopus, International Pharmaceutical Abstract, Chemical Abstracts, Embase, Index Copernicus, EBSCO, African Index Medicus, JournalSeek, Journal Citation Reports/Science Edition, Directory of Open Access Journals (DOAJ), African Journal Online, Bioline International, Open-J-Gate and Pharmacy Abstracts

\section{INTRODUCTION}

Anxiety disorder as the most prevalent mental symptom [1], it is caused by genetic, mental and social factors, and manifested in various fancies and conjectures, restlessness and abnormal suffering, which can cause fear and even panic to a certain extent. Anxiety disorder is due to disproportionate activation of the sympathetic and parasympathetic systems, with obvious influence on the cardiovascular system. The main cardiovascular manifestations are 
palpitation, increased heartbeat, chest pain, dizziness, and numbness. In the acute coronary syndrome population, anxiety disorder leads to a high risk of subsequent myocardial infarction [2].

Since the emergence of coronavirus disease in 2019 (COVID-19), more than 100 million people have been infected worldwide (as at February 2021). The COVID-19 pandemic has led to increased economic pressure and stress due to death, leading to increases in anxiety disorders. Therefore, it is necessary to find more effective ways of treating anxiety disorders.

At present, there are two ways of treating anxiety disorder: psychological treatment, based on cognitive behavioral therapy, computer-assisted and internet-based treatments; and pharmacological treatments using certain inhibitors which would involve in the anxiety pathway, such as serotonin-reuptake inhibitors, serotonin-noradrenaline-reuptake inhibitors, tricyclic antidepressants and monoamine oxidase inhibitors. These treatments are $60-70 \%$ effective [1]. However, the associated side effects such as withdrawal symptoms, safety problems, complexity of medication, and high costs have limited the clinical application of psychological and pharmacological treatments. Therefore, it is of great significance to seek treatment methods or drugs with little or no side effects.

Essential oils regulate various mental discomforts through their fragrance. Different essential oils have different therapeutic effects [3]. AEO is a volatile oil extracted from Angelica sinensis. It is composed of small-molecule compounds with the unique aroma of Angelica sinensis. Studies have shown that certain concentrations of AEO exhibited obvious antianxiety effects in male Swiss mice [4]. Some research revealed that $\mathrm{AEO}$ has potential usefulness on disorders caused by anxiety and social inadequacy [5]. Therefore, AEO is a novel therapeutic approach to anxiety disorders, However, the pharmacological mechanism underlying its effect has not been elucidated.

In this study, the pharmacological mechanism of which AEO on anxiety disorders was uncovered using network pharmacology, and verified via molecular docking. The compositions of AEO were determined using GC-MS. The bioinformatics databases were explored to get the targets of AEO on anxiety disorders. Cytoscape software was applied to discover the relationship of component with target, protein with protein and target with tissue distribution, while $\mathrm{R}$ was applied to perform the $\mathrm{GO}$ and conduct KEGG pathway of the targets. Autodock vina software was applied to figure out the interaction between the main active chemical components and protein targets.

\section{EXPERIMENTAL}

\section{Identification of chemical components and screening of targets}

The components of AEO were recognized using GC-MS. The gas chromatographic requirements were as follows: capillary column was Agilent HP-5 $(0.25 \mathrm{~mm} \times 30 \mathrm{~m}, 0.25 \mu \mathrm{m})$; the carrier gas was Helium (99.999\%), the sample volume used was $1 \mu \mathrm{L}$, the shunt ratio was $40: 1$, while the flow rate was $1 \mathrm{~mL} / \mathrm{min}$. The temperature was programmed viz: initial temperature of $80{ }^{\circ} \mathrm{C}$ with a heating rate of $3{ }^{\circ} \mathrm{C} / \mathrm{min}$ up to $167^{\circ} \mathrm{C}$ in $2.5 \mathrm{~min}$; and thereafter at $2{ }^{\circ} \mathrm{C} / \mathrm{min}$ up to $202{ }^{\circ} \mathrm{C}$, followed by $4{ }^{\circ} \mathrm{C} / \mathrm{min}$ up to $260{ }^{\circ} \mathrm{Cin} 2 \mathrm{~min}$. The mass spectrometry requirements were: $\mathrm{El}$ ion source, electron energy of $70 \mathrm{eV}$, temperature of ion source and MS quadrupole were $230{ }^{\circ} \mathrm{C}$ and 150 ${ }^{\circ} \mathrm{C}$, respectively; $3.0 \mathrm{~min}$ solvent delay, and full scan pattern. Chemical components with quality greater than 80 were screened with Database of SwissTargetPrediction [6] (http://swisstargetp rediction.ch) and TCMSP [7] (http://lsp.nwu. edu.cn/tcmsp.php) to gain the targets.

\section{Screening for anxiety targets}

The TTD [8] (https://db.idrblab.org/ttd/), DrugBank [9] (https://www.drugbank.ca/) and DisGeNET [10] (http://www.disgenet.org/) databases were searched for anxiety targets.

\section{Networking the interaction of bioactive component and target}

The intersection of the chemical component targets and anxiety targets were taken as the potential targets, and the chemical components corresponding to the potential targets were considered as the potential bioactive components [11]. UniProt database [12] (http://www.uniprot.org/) was utilized to obtain the UniProt ID of targets. The bioactive components and potential targets were imported into Cytoscape 3.7.1 software so as to network the bioactive component and potential target.

\section{Networking and analysis of the interaction of potential targets}

The potential target was imported into the STRING database [13] (https://string-db.org/); the species was defined as human, and the result was saved in the TSV format. Then, the 
information on the interaction of protein and protein was analyzed by Cytoscape 3.7.1 software.

\section{Networking and analysis of the tissue distribution and targets network}

Human

Protein

Atlas

(https://www.proteinatlas.org/) was searched for tissue data on potential targets. Tissues corresponding to expression values (NX) greater than 5 in the data were analyzed using Cytoscape 3.7.1 software in order to get the tissue distributions of potential targets.

\section{GO and KEGG pathway analysis}

Analyses of GO (including $\mathrm{BP}, \mathrm{MF}$ and $\mathrm{CC}$ ) and KEGG pathway were done using clusterProfiler(R). This served to reveal the pharmacological mechanism with the effect of AEO on anxiety.

\section{Molecular docking of the main bioactive components on targets}

Autodock vina1.1.2 software was used to determine the interaction/binding between the main bioactive chemical components of $A E O$ and protein targets. The structure of protein targets and main bioactive chemical components were uploaded through PDB database [15] (www.rcsb.org/) and PubChem Database [16] (https://pubchem.ncbi.nlm.nih.gov/) respectively, and the molecular docking analysis was conducted.

\section{RESULTS}

\section{Chemical components in AEO}

In the results from GC-MS (Figure 1), a total of 31 chemical components of AEO were identified via searching the database NIST14.L in Agilent 7890A/5975C. Chemical components with quality value greater than 80 are shown in Table 1. Cisligustilide was the major component of the $\mathrm{AEO}$, accounting for an area percentage of $78.96 \%$, relative to the other 10 components.

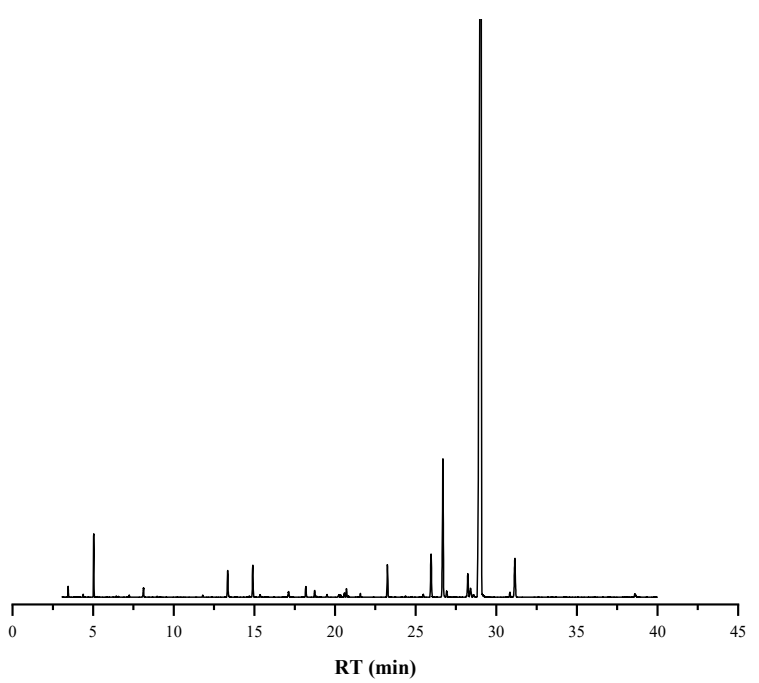

Figure 1: GC-MS chromatogram of AEO

\section{Acquisition of potential targets}

The Targets of 11 chemical components were searched via the TCMSP and SwissTargetPrediction. Anxiety-related targets were obtained using the TTD, DrugBank and DisGeNET. 34 potential targets (Table 2) were acquired, from the intersection targets of chemical components targets and anxiety targets.

\section{Networking of bioactive component and action target}

The component - target network (Figure 2) was constructed. There were 44 nodes in the network, including 10 compound nodes (transbeta-ocimene was not associated and removed) and 34 target nodes.

Table 1: Information of AEO components

\begin{tabular}{llllcc}
\hline No. & Retention time $(\min )$ & Area $(\%)$ & Components & CAS & Quality \\
\hline 1 & 3.4518 & 0.3941 & Alpha-pinene & $000080-56-8$ & 95 \\
2 & 5.0372 & 2.0762 & Trans-beta-Ocimene & $003779-61-1$ & 97 \\
3 & 13.3462 & 0.7718 & 2-Methoxy-4-vinylphenol & $007786-61-0$ & 90 \\
4 & 17.1224 & 0.3317 & Germacrene D & $023986-74-5$ & 87 \\
5 & 23.2552 & 1.3232 & Espatulenol & $006750-60-3$ & 96 \\
6 & 26.6983 & 6.4862 & 3-butylidenephthalide & $000551-08-6$ & 96 \\
7 & 29.0728 & 78.9609 & Cis-ligustilide & $1000365-98-5$ & 91 \\
8 & 31.1631 & 1.9236 & Trans-ligustilide & $1000365-98-8$ & 94 \\
9 & 38.6207 & 0.4373 & N-Hexadecanoic acid & $000057-10-3$ & 95 \\
10 & 44.3068 & 0.1579 & Methyl linolelaidate & $002462-85-3$ & 87 \\
11 & 45.9618 & 0.2560 & Linoleic & $000060-33-3$ & 94 \\
\hline
\end{tabular}


Table 2: Profile of potential targets

\begin{tabular}{|c|c|c|c|c|}
\hline S/no. & Gene & Protein & UniProt ID & Degree \\
\hline 1 & CYP2C19 & Cytochrome P450 2C19 & P33261 & 1 \\
\hline 2 & $\mathrm{BCHE}$ & Butyrylcholine esterase & P06276 & 1 \\
\hline 3 & CYP1A2 & Cytochrome P450 1A2 & P05177 & 1 \\
\hline 4 & CYP1A1 & Cytochrome P450 1A1 & P04798 & 1 \\
\hline 5 & ESR1 & Estrogen receptor & P03372 & 2 \\
\hline 6 & DAO & D-amino-acid oxidase & P14920 & 1 \\
\hline 7 & ADRA1B & Alpha-1B adrenergic receptor & P35368 & 2 \\
\hline 8 & GABRA1 & Gamma-aminobutyric acid receptor subunit alpha-1 & P14867 & 4 \\
\hline 9 & CYP17A1 & Cytochrome P450-C17 & P05093 & 1 \\
\hline 10 & GABRB2 & Gamma-aminobutyric acid receptor subunit beta- 2 & P47870 & 3 \\
\hline 11 & PDE10A & $\begin{array}{l}\text { CAMP and cAMP-inhibited cGMP } 3^{\prime}, 5^{\prime} \text {-cyclic } \\
\text { phosphodiesterase } 10 \mathrm{~A}\end{array}$ & Q9Y233 & 3 \\
\hline 12 & ADRA1A & Alpha- $1 \mathrm{~A}$ adrenergic receptor & P35348 & 1 \\
\hline 13 & GABRB3 & Gamma-aminobutyric acid receptor subunit beta- 3 & P28472 & 3 \\
\hline 14 & GABRG2 & Gamma-aminobutyric acid receptor subunit gamma-2 & P18507 & 3 \\
\hline 15 & MAOA & Monoamine oxidase type A & P21397 & 4 \\
\hline 16 & KCNH2 & Potassium voltage-gated channel subfamily $\mathrm{H}$ member 2 & Q12809 & 1 \\
\hline 17 & CYP2A6 & Cytochrome P450 2A6 & P11509 & 3 \\
\hline 18 & IL10 & Interleukin-10 & P22301 & 1 \\
\hline 19 & SLC6A2 & Sodium-dependent noradrenaline transporter & P23975 & 2 \\
\hline 20 & SLC6A4 & Sodium-dependent serotonin transporter & P31645 & 5 \\
\hline 21 & PTGS1 & Prostaglandin G/H synthase 1 & P23219 & 5 \\
\hline 22 & CNR1 & Cannabinoid receptor 1 & P21554 & 2 \\
\hline 23 & PTGS2 & Prostaglandin G/H synthase 2 & P35354 & 6 \\
\hline 24 & SLC6A3 & Sodium-dependent dopamine transporter & Q01959 & 4 \\
\hline 25 & $\mathrm{FAAH}$ & Fatty-acid amide hydrolase 1 & O00519 & 4 \\
\hline 26 & ADRA2A & Alpha-2A adrenergic receptor & P08913 & 5 \\
\hline 27 & ADRA2B & Alpha-2B adrenergic receptor & P18089 & 5 \\
\hline 28 & ADRA2C & Alpha-2C adrenergic receptor & P18825 & 3 \\
\hline 29 & MAPT & Microtubule-associated protein tau & P10636 & 2 \\
\hline 30 & CHRM2 & Muscarinic acetylcholine receptor M2 & P08172 & 8 \\
\hline 31 & CHRM4 & Muscarinic acetylcholine receptor M4 & P08173 & 4 \\
\hline 32 & CHRM5 & Muscarinic acetylcholine receptor M5 & P08912 & 4 \\
\hline 33 & CHRM1 & Muscarinic acetylcholine receptor M1 & P11229 & 7 \\
\hline 34 & CHRM3 & Muscarinic acetylcholine receptor M3 & P20309 & 5 \\
\hline
\end{tabular}

The size of the nodes represented the degree value, and the "degree" represented the action intensity. It had 107 edges, and each edge represented the relationship between a compound and target. The dark blue $\mathrm{V}$ nodes represented the bioactive component, while the green ellipse nodes represented the potential target. In the entire network, the chemical components 3-butylidenephthalide, transligustilide and cis-ligustilide were highly correlated with potential targets, with degree values were $20,19,19$ respectively. The targets CHRM2, CHRM1 and PTGS2 were highly correlated with the chemical components, and the degree values were $8,7,6$ respectively.

\section{Networking and analysis of protein-protein interaction networks}

Figure 3 shows the constructed network of protein-protein interactions (PPI) of the 34 potential targets. The PPI network contained 33 node proteins (one protein target was not associated, and so was removed) and 104 interaction lines.

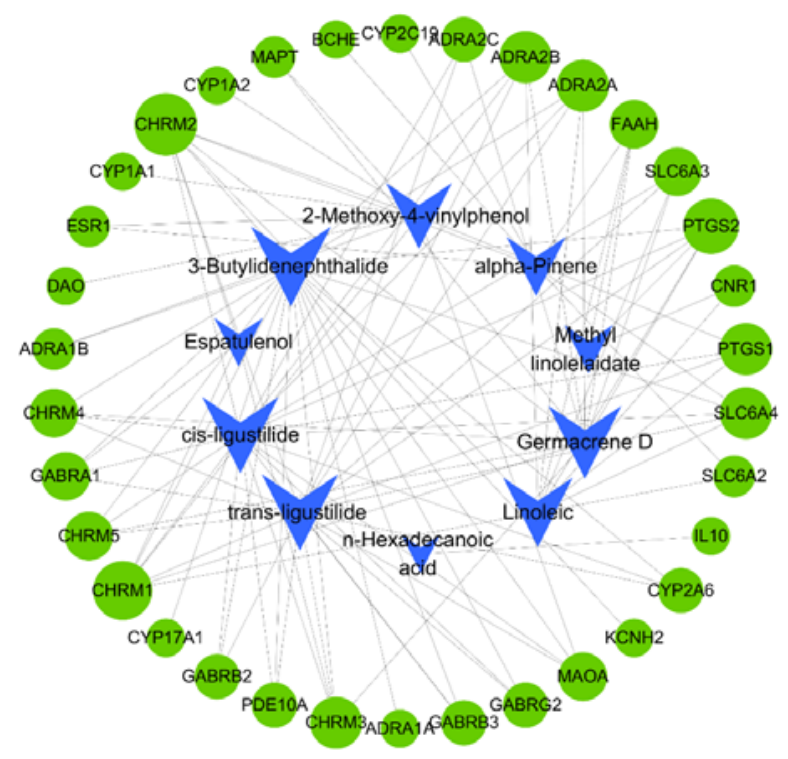

Figure 2: Component-target network of AEO (components of dark blue $\mathrm{V}$ nodes were connected to targets in green ellipse nodes) 
The node size represented the degree value. In the protein-protein interaction network, the targets with degree exceeding 10 were SLC6A4 (degree 14), MAOA (degree 13), CNR1 (degree 10), ADRA2A (degree 10) and ADRA2C (degree 10).

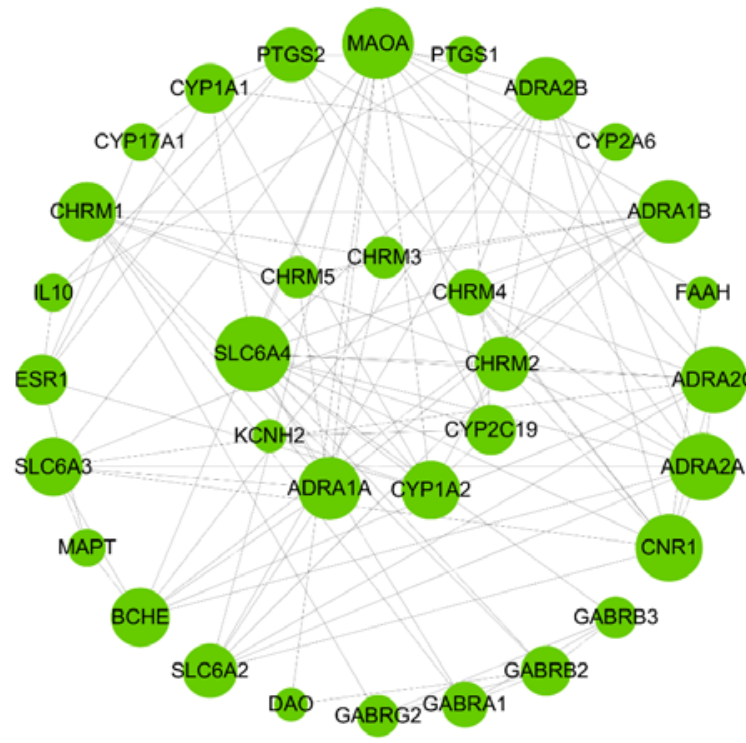

Figure 3: Protein-protein interaction network of potential targets

\section{Networking and analysis of tissue distribution and potential targets}

The network of tissue distribution - potential targets was constructed through The Human Protein Atlas database and Cytoscape 3.2.1. The network is shown in Figure 4. After removing the unrelated nodes, there were 90 nodes and 380 edges (the green ellipse nodes represented potential targets, the blue round rectangle nodes represented organ or tissue, while the node size represented the degree value). Each edge represented the relationship between the potential target and tissue or organ. The network analysis results showed that 34 targets were distributed in the cerebellar cortex (degree 16), basic ganglia (degree 15), hippocampal formation (degree 12), amygdala (degree 12), pons and medulla (degree 12), and liver (degree 12). In particular, MAOA (degree 45) and FAAH (degree 43) were the targets most closely related to organ or tissue.

\section{GO and KEGG pathway analysis}

GO and KEGG pathway analysis ( $p \leq 0.05$ ) of potential targets were carried out via ClusterProfiler (R). In the results of $\mathrm{GO}$ analysis, there were 294 items (BP) that were related to response to ammonium ion, adenylate cyclase- modulating G-protein-coupled receptor signaling pathway, and G-protein-coupled receptor signaling pathway coupled to cyclic nucleotide second messenger. There were $47 \mathrm{CC}$ items that involve synaptic membrane and postsynaptic membrane. There were 85 items in MF that involved G-protein-coupled amine receptor activity and neurotransmitter receptor activity. As indicated in Figure 5, the first 10 items in analyse of BP, CC and MF are shown.

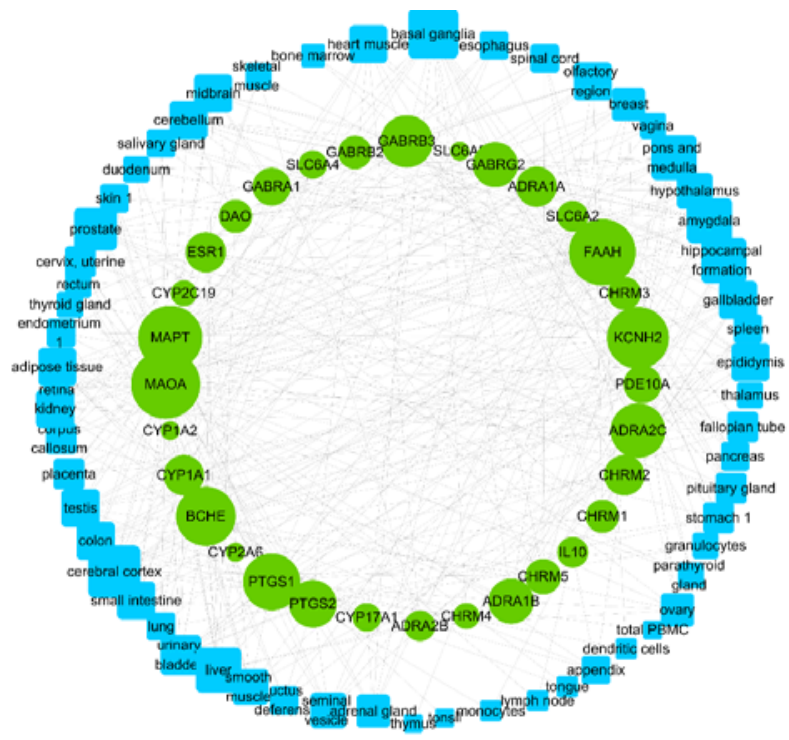

Figure 4: Tissue distribution - potential targets network (targets in green ellipse nodes were connected to tissues or organs in blue round rectangle nodes)

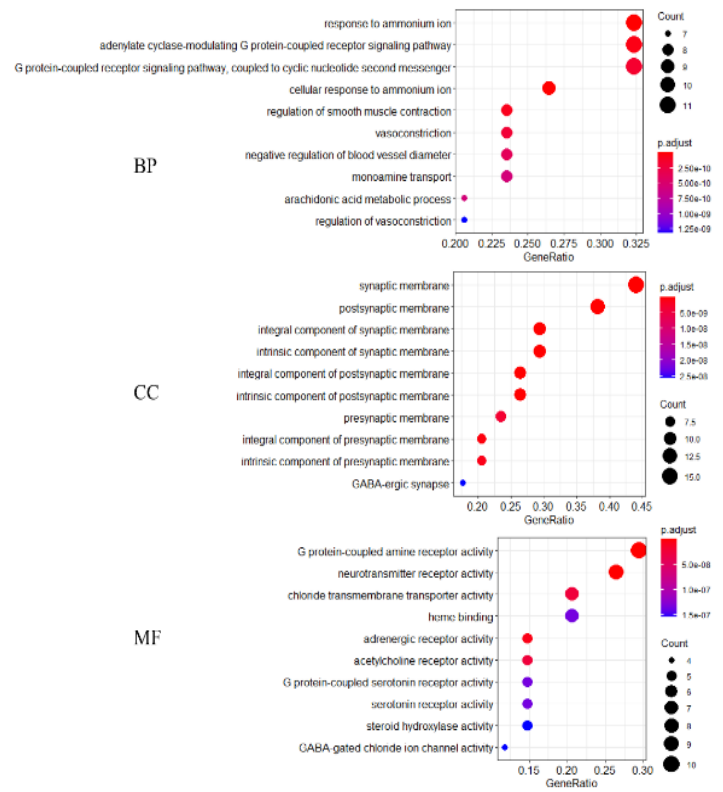

Figure 5: Bubble diagram of Gene Ontology enrichment analysis

Trop J Pharm Res, April 2021; 20(4): 793 
KEGG enrichment analysis mapped 22 signal pathways. The first 10item pathways are shown in Figure 6. The main pathway was neuroactive ligand-receptor interaction.

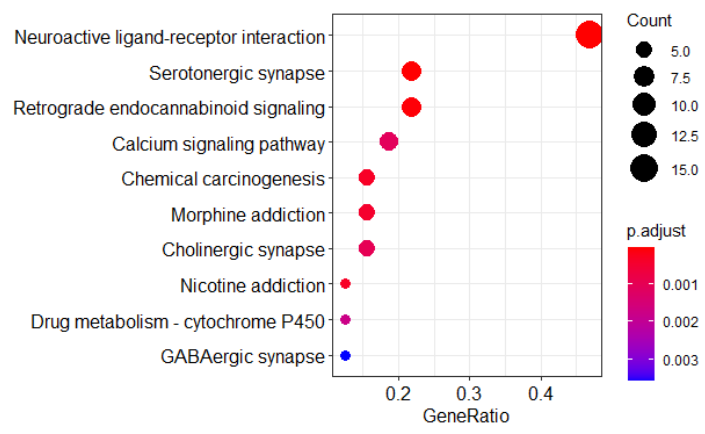

Figure 6: Bubble diagram of KEGG enrichment analysis

\section{Molecular docking of main active ingredients to targets}

The unique ligands of the main active targets of CHRM2, CHRM1 and PTGS2 in PDB database were glycerol, methscopolamine and 1,2ethanediol, respectively. The binding abilities of the main active targets to glycerol, methscopolamine, and 1,2-ethanediol (as control) were compared with the binding abilities of the main active targets to the main bioactive components (3-butylidenephthalide, transligustilide and cis-ligustilide. The binding energies of ligands (control and three active ingredients) to the receptor (the active targets) were analyzed using Autodock vina1.1.2 software. The binding energy (affinity value) results are listed in Table 3 . The smaller the binding energy, the better the binding ability. Compared with the control, the main active components showed good binding ability. Transligustilide and cis-ligustilide are isomers of one another and 3-butylidenephthalide has a similar chemical structure to the two isomers (transligustilide and cis-ligustilide). Thus, they had the same binding energy.

\section{DISCUSSION}

Studies have shown that AEO exerts an antianxiety effect. This work applied network pharmacology to systematically determine the pharmacological mechanism involved in the effect of AEO on anxiety, at the molecular level. In GC-MS test, eleven chemical components were selected for analysis, with cis-ligustilide being the most abundant component. In component-target network, the main bioactive components were 3-butylidenephthalide, transligustilide and cis-ligustilide, while the main active targets were CHRM2, CHRM1, PTGS2. The three main bioactive components have sedative, anti-spasmodic, anti-asthmatic and strong pharmacological effects on cardiocerebrovascular and immune systems $[17,18]$. It has been revealed that $\mathrm{CHRM} 2$ is muscarinic acetylcholine receptor $\mathrm{M} 2$ involved in neuronal excitability, advanced cognitive processing, and memory and cognitive impairment in many neuropsychiatric disorders $[19,20]$.

Muscarinic acetylcholine receptor M1 (CHRM1) improves cognitive deficits in schizophrenia [21]. Prostaglandin G/H synthase 2 (PTGS2) inhibits apoptosis and immune surveillance, and enhances angiogenesis [22]. Anxiety is caused by excessive activation of nervous system, and often occurs in cognitive impairment [2]. Long term anxiety affects the cardiovascular and immune systems. Therefore, AEO may exert sedative, neuroprotective and immune-regulating effects in anxiety disorder through interaction with its active targets.

From the PPI network of screening 34 potential targets, the core targets were SLC6A4, MAOA, CNR1, ADRA2A and ADRA2C.Sodiumdependent serotonin transporter (SLC6A4) is a serotonin transporter gene. Study has shown that SLC6A4 is associated with the etiology of anxiety disorder [23]. Monoamine oxidase type A (MAOA) gene is associated with emotional stability and neuroticism [24].

Through analysis of tissue distribution/potential targets network, it was revealed that the potential targets were mainly distributed in the cerebellar cortex, basic ganglia, hippocampal formation, amygdala, pons and medulla, and liver. This result is consistent with that the fact that the major areas involved in anxiety reactions are the cerebral cortex, hippocampus and amygdala.

Table 3: Analysis results of the optimal binding in Autodock vina

\begin{tabular}{lcccc}
\hline Active & \multicolumn{4}{c}{ Binding energy (kcal/mol) } \\
\cline { 2 - 5 } target & Control & \multicolumn{3}{c}{ Active componet } \\
\hline CHRM1 & Glycerol & Trans-ligustilide & Cis-ligustilide & 3-butylidenephthalide \\
& -3.5 & -6.0 & -6.0 & -6.0 \\
CHRM2 & Methscopolamine & Trans-ligustilide & Cis-ligustilide & 3-butylidenephthalide \\
PTGS2 & -6.0 & -6.0 & -6.0 & -6.0 \\
\hline
\end{tabular}


From KEGG pathway results, neuroactive ligandreceptor interaction was the main pathway through which AEO affected anxiety.

Autodock Vina software has the advantages of speed and accuracy in analysis of molecular docking. This study compared the binding capacities of the controls to the main active targets, with those of the main bioactive components in Autodock Vina. The results showed that the bioactive components had the same or lower binding energies with the controls, which indicated that the bioactive components had good binding ability to the active targets.

\section{CONCLUSION}

The pharmacological mechanism involved in the effect of AEO on anxiety has systematically been explored using network pharmacology. The analysis has identified 10 bioactive components and 34 potential targets. The results obtained suggest that AEO acts on anxiety through multicomponent, multi-target, multi-channel and multiorganization pathways. Molecular docking reveals low binding energies of the bioactive components to their protein targets. Thus, this study provides a theoretical basis for further studies on the effect of AEO on anxiety.

\section{DECLARATIONS}

\section{Acknowledgement}

The authors appreciate the assistance of Innovative Research Team Project of Chinese Medicine Discipline in Chengdu University of Traditional Chinese Medicine (no. CXTD2018006) and Major $R$ \& D projects of Jiangxi Province (no. 20194ABC28009).

\section{Conflict of interest}

No conflict of interest is associated with this study.

\section{Contribution of authors}

We declare that this work was done by the authors named in this article, and all liabilities pertaining to claims related to the content of this article are borne by the authors. Xiaoli Liu designed the study and analyzed the data. Xiaofei Zhang interpreted the data; Fang Wang prepared the manuscript for publication. Jie $\mathrm{Xu}$ supervised the data collection, while Ming Yang reviewed the draft of the manuscript. All authors read and approved the manuscript.

\section{Open Access}

This is an Open Access article that uses a funding model which does not charge readers or their institutions for access and distributed under the terms of the Creative Commons Attribution License (http://creativecommons.org/licenses/by/ 4.0) and the Budapest Open Access Initiative (http://www.budapestopenaccessinitiative.org/rea d), which permit unrestricted use, distribution, and reproduction in any medium, provided the original work is properly credited.

\section{REFERENCES}

1. Craske MG, Stein MB. Anxiety. Lancet 2016; 388: 30483059.

2. Tully PJ, Harrison NJ, Cheung P, Cosh S. Anxiety and Cardiovascular Disease Risk: a Review. Curr Cardiol Rep 2016; 18:120.

3. Zhang N, Yao L. Anxiolytic Effect of Essential Oils and Their Constituents: A Review. J Agric Food Chem 2019; 1-19.

4. Chen SW, Li Min, Li WJ, Kong WX, Li JF, Zhang YJ. The effects of angelica essential oil in three murine tests of anxiety. Pharmacol Biochem Be 2004; 79: 377-382.

5. Min L, Chen SW, Li WJ, Wang R, Li YL, Wang WJ, Mi $X J$. The effects of angelica essential oil in social interaction and hole-board tests. Pharmacol Biochem Be 2005; 81: 838-842.

6. Daina A, Michielin $O$, Zoete $V$. SwissTargetPrediction: updated data and new features for efficient prediction of protein targets of small molecules, Nucl. Acids Res.2019,47(W1): W357-W364.

7. Ru J, Li P, Wang J, Zhou W, Li BH, Huang C, Li PD, Guo $Z H$, Tao WY, Yang YF, et al. TCMSP: a database of systems pharmacology for drug discovery from herbal medicines. J Cheminform 2014; 6: 13.

8. Li YH, YU CY, Li XX, Zhang P, Tang J, Yang QX, Fu TT, Zhang $X Y$, Cui $X J$, Tu $G$, et al. Therapeutic target database update 2018: enriched resource for facilitating bench to clinic research of targeted therapeutics. Nucleic Acids Res 2018; 46(D1): D1121-D1127.

9. Wishart DS, Feunang YD, Guo AC, Lo EJ, Marcu A, Grant JR, Sajed T, Johnson D, Li C, Sayeeda Z, et al. DrugBank 5.0: a major update to the DrugBank database for 2018.Nucleic Acids Res 2018; 46(D1): D1074-D1082.

10. Piñero J, Bravo À, Queralt-Rosinach N, GutiérrezSacristán A, Deu-Pons J, Centeno E, García-García J, Sanz F, Furlong LI. DisGeNET: a discovery platform for the dynamical exploration of human diseases and their genes. Database (Oxford) 2015: bav028.

Trop J Pharm Res, April 2021; 20(4): 795 
11. Guilin Ren, Yu Zhong, Gang Ke, Xiaoli Liu, Huiting Li, Xiaofang Li, Qin Zheng, Ming Yang. The Mechanism of Compound Anshen Essential Oil in the Treatment of Insomnia was Examined by Network Pharmacology. EVID-BASED COMPL ALT 2019;1-9.

12. The UniProt Consortium. UniProt: the universal protein knowledgebase. Nucleic Acids Res. 2017;45(D1): D158D169.

13. Mering $C$, Huynen $M$, Jaeggi $D$, Schmidt $S$, Bork $P$, Snel B. STRING: a database of predicted functional associations between proteins. Nucleic Acids Res 2003; 31(1): 258- 261.

14. Thul PJ, Lindskog $C$. The human protein atlas: $A$ spatial map of the human proteome. Protein Sci 2018; 27(1): 233-244.

15. Berman HM, Westbrook J, Feng Z, Gilliland G, Bhat TN, Weissig $H$, Shindyalov IN, Bourne PE, et al. The Protein Data Bank. Nucleic Acids Res 2000; 28(1): 235-242. doi:10.1093/nar/28.1.235

16. Kim S, Thiessen PA, Bolton EE, Chen J, Fu G, Gindulyte $A$, Han $L Y$, He J, He SQ, Shoemaker BA, et al. PubChem Substance and Compound databases. Nucleic Acids Res 2016; 44(D1): D1202-D1213.

17. Zhu MD, Zhao LX, Wang $X T$, Gao YJ, Zhang $Z J$. Ligustilide inhibits microglia-mediated proinflammatory cytokines production and inflammatory pain. Brain Res Bull 2014; 109: 54-60.

18. Long $F Y$, Shi MQ, Zhou HJ, Liu DL, Sang N, Du JR. Klotho upregulation contributes to the neuroprotection of ligustilide against cerebral ischemic injury in mice. Eur $\mathrm{J}$ Pharmacol 2018; 820: 198-205.

19. Zink N, Bensmann W, Arning L, Stock AK, Beste C. CHRM2 Genotype Affects Inhibitory Control Mechanisms During Cognitive Flexibility. Mol Neurobiol 2019.

20. Luo XG, Kranzler HR, Zuo LJ, Wang S, Blumberg HP, Gelernter J. CHRM2 gene predisposes to alcohol dependence, drug dependence and affective disorders: results from an extended case-control structured association study. Hum Mol Genet 2005; 14: $2421-$ 2434.

21. Scarr E, Sundram S, Deljo A, Cowie TF, Gibbons AS, Juzva S, Mackinnon A, Wood SJ, Testa R, Pantelis C, et al. Muscarinic M1 receptor sequence: Preliminary studies on its effects on cognition and expression. Schizophr Res 2012; 138: 94-98.

22. Seo MJ, Oh DK. Determinants of the cellular specificity of acetaminophen as an inhibitor of prostaglandin $\mathrm{H} 2$ synthases. P Natl A Sci 2002; 99: 7130-7135.

23. Forstner AJ, Rambau S, Friedrich N, Ludwig KU, Böhmer AC, Mangold E, Maaser A, Hess T, Kleiman A, Bittner $A$, et al. Further evidence for genetic variation at the serotonin transporter gene SLC6A4 contributing toward anxiety. Psychiat Genet 2017; 27: 96-102.

24. Rodríguez-Ramos Á, Moriana JA, García-Torres F, Ruiz-Rubio M. Emotional stability is associated with the MAOA promoter UVNTR polymorphism in women. Brain Behav 2019; 00: 1-12. 